\title{
UM BREVE ESTUDO DOS PARÂMETROS DO MÉTODO ENXAME DE PARTÍCULAS
}

\author{
Shayane da Silva Carvalho \\ Universidade Federal Fluminense/INFES \\ Santo Antônio de Pádua, RJ \\ shay-carvalho@hotmail.com \\ Joviana Sartori de Souza \\ Universidade Federal Fluminense/INFES \\ Santo Antônio de Pádua, RJ \\ joviana.sartori@gmail.com
}

\begin{abstract}
RESUMO
Este artigo tem como finalidade apresentar uma análise de uma metaheurística populacional, o Enxame de Partículas (PSO), aplicado na resolução de um problema termodinâmico. O problema do cálculo do equilíbrio de fases de uma mistura está presente em processos da Engenharia Química. Para ser resolvido é necessário que se conheça a priori o número de fases presentes na mistura, para isto deve-se solucionar o problema de otimização conhecido como teste de estabilidade. O objetivo central do trabalho é aplicar o método de busca global na minimização do problema objetivo. Será utilizada a técnica da polarização que permite encontrar todos os pontos estacionários da função, e também serão avaliadas estratégias de valoração dos parâmetros do PSO. Os resultados numéricos dos testes serão apresentados para três misturas binárias já abordadas na literatura, de forma a avaliar qual metodologia proposta será a mais robusta para eficiência computacional do método.
\end{abstract}

Palavra-chave: Otimização; Enxame de Partículas; Problema Termodinâmico; Teste de Estabilidade.

\begin{abstract}
This article aims to present an analysis of a population metaheuristic, the Particle Swarm (PSO), applied to solve a thermodynamic problem. The problem of calculating the phase equilibrium of a mixture is present in Chemical Engineering processes. To be solved it is necessary to know a priori the number of phases present in the mixture, for this one must solve the optimization problem known as stability test. The main objective of this work is to apply the global search method to minimize the objective problem. It will be used the polarization technique that allows to find all the stationary points of the function, and will also be evaluated strategies of PSO parameter valuation. The numerical results of the tests will be presented for three binary mixtures already discussed in the literature, in order to evaluate which proposed methodology will be the most robust for the computational efficiency of the method.
\end{abstract}

Keywords: Optimization; Particle Swarm; Thermodynamic problem; Stability Test. 


\section{Como Citar:}

CARVALHO, Shayane da Silva; SOUZA, Joviana Sartori de. Um breve estudo dos parâmetros do método Enxame de Partículas. In: SIMPÓSIO DE PESQUISA OPERACIONAL E LOGÍSTICA DA MARINHA, 19., 2019, Rio de Janeiro, RJ. Anais [...]. Rio de Janeiro: Centro de Análises de Sistemas Navais, 2019.

\section{INTRODUÇÃO}

O problema abordado neste artigo se enquadra em um tema de grande importância em processos da Engenharia Química: o cálculo do equilíbrio de fases. Este é um problema de otimização conhecido como a minimização da função distância do plano tangente à energia livre de Gibbs molar. Para ser resolvido é necessário que se faça uma análise prévia da estabilidade termodinâmica do sistema.

Como enfatizado por Michelsen (1982), Sun et al. (1995), Stadtherr et al. (1995) e por Lucia et al. (2005), para proporcionar uma completa predição do equilíbrio de fases, fazse necessário não apenas a determinação do minimizador global da função objetivo do teste de estabilidade mas também a obtenção de todos os seus pontos estacionários.

Neste trabalho realiza-se uma análise no método estocástico Enxame de Partículas (PSO) aplicado ao processo de minimização do problema termodinâmico que é descrito por modelos de natureza não convexa e não linear.

O objetivo central deste artigo é comparar cinco estratégias de valoração dos parâmetros do método PSO e verificar a partir dos resultados obtidos, qual metodologia será a mais robusta na determinação dos pontos estacionários do problema proposto. Assim, pode-se analisar a influência dos valores de parâmetros no desempenho do método.

\section{O PROBLEMA DO TESTE DE ESTABILIDADE}

O presente estudo aborda apenas o teste de estabilidade de fases básico de uma mistura onde procura-se analisar se um sistema termodinâmico composto por diversos componentes químicos apresenta-se em uma fase simples ou mais fases. Trata-se da relação com a transferência de massa entre subsistemas de um sistema composto.

As condições de equilíbrio de um sistema em que são conhecidas as composições de cada um dos componentes químicos, a temperatura e a pressão, podem ser atingidas quando a função distância do plano tangente à energia livre de Gibbs é minimizada.

Considerando uma mistura que se encontra a temperatura $T$, pressão $P$ e possui $r$ componentes químicos representados pelos seus números de moles $N_{1}, \ldots, N_{r}$. Com o intuito de se estudar a estabilidade desta mistura em relação ao processo de transferência de massa a $T$ e $P$ constantes, suponha inicialmente que tal mistura encontra-se numa fase simples a qual será chamada de fase inicial. Sabe-se que a energia livre de Gibbs do sistema nestas condições de fase simples é dada por

$$
G_{0} \equiv G\left(N_{1}, \ldots, N r\right)=\sum_{i=1}^{r} N_{i} \mu_{i}(0)
$$

Sabe-se também que o potencial químico de cada componente químico na fase simples é uma função homogênea de grau zero e, portanto, pode ser escrito na forma 


$$
\mu_{i}=\mu_{i}\left(N_{1}, \ldots, N_{r}\right)=\mu_{i}\left(z_{1}, \ldots, z_{r}\right) \cdot(0)
$$

Na equação (2), $z_{i}=N_{i} / \Sigma_{i=1}^{r} N_{i}$ é a fração molar global do componente $i(i=1, \ldots$, r) na mistura multicomponente. Desta forma, por definição as frações molares satisfazem à restrição $\sum_{i=1}^{r} z_{i}=1$.

A função objetivo a ser minimizada denominada função distância do plano tangente à energia livre de Gibbs molar pode ser definida como:

$$
d\left(x_{1}, \ldots, x_{r}\right)=\sum_{i=1}^{r} x_{i}\left[\mu_{i}\left(x_{1}, \ldots, x_{r}\right)-\mu_{i}\left(z_{1}, \ldots, z_{r}\right)\right](0)
$$

para todo $\left(x_{1}, \ldots, x_{r}\right)^{\mathrm{T}}$ pertencente ao conjunto

$$
\Omega=\left\{\left(x_{1}, \ldots, x_{r}\right)^{T} \in R^{r} ; 0<x_{i}<1 ; \forall i=1, \ldots, r e \sum_{i=1}^{r} x_{i}\right\} \cdot(0)
$$

Onde $x_{i}$ é a fração molar do componente $i$ presente na mistura, $z_{i}$ é a composição global do componente $i$ na mistura, $\mu_{i}$ é o potencial químico do componente $i$.

O critério de estabilidade pode ser realizado através da função $d$ : se $d\left(x_{1}, \ldots, x_{r}\right) \geq 0$, para todo $\left(x_{1}, \ldots, x_{r}\right)^{\mathrm{T}}$ em $\Omega$ a mistura com componentes globais $z_{1}, \ldots, z_{r}$ é estável e permanecerá no estado homogêneo inicial, nas referidas temperatura e pressão. Caso contrário, a mistura é instável e deve se dividir em duas ou mais fases.

Uma possível maneira de implementar um teste de estabilidade baseado no critério do plano tangente de Gibbs consiste em resolver o problema de otimização global descrito a seguir.

$$
\left\{\begin{array}{l}
\text { Dados } z=\left(z_{1}, \ldots, z_{r}\right)^{t} \in \Omega, T_{0} e P_{0} z=\left(z_{1}, \ldots, z_{r}\right)^{t} \in \Omega, T_{0} e P_{0} z=\left(z_{1}, \ldots, z_{r}\right)^{T} \in \Omega, T_{0} e P_{0}, \\
\text { Encontrar } x=\left(x_{i} i 1, \ldots, x_{r}\right)^{T} \in R^{r} i \text { a fim de } \\
\text { Minimizar } d\left(T_{0}, P_{0}, x_{1}, \ldots, x_{r}\right)=\sum_{i=1}^{r} x_{i}\left[\mu_{i}\left(T_{0}, P_{0}, x_{1}, \ldots, x_{r}\right)-\mu_{i}\left(T_{0}, P_{0}, z_{1}, \ldots, z_{r}\right)\right]
\end{array}\right.
$$

Sujeito à seguinte restrição:

$$
x \in \Omega=\left\{\left(x_{1}, \ldots, x_{r}\right)^{T} \in R^{r} ; 0<x_{i}<1, \forall i=1, \ldots, r e \sum_{i=1}^{r} x_{i}=1\right\}
$$

Se $x^{i} \in \Omega$ é um minimizador global do problema (P1), têm-se que $d\left(T_{0}, P_{0}, x^{i}\right) \leq d\left(T_{0}, P_{0}, x\right)$, para todo $x \in \Omega$. Sabe-se que o ponto $x$ é denominado ponto estacionário da função $d$ se $\nabla d(x)=0$. Dentre esses pontos estacionários encontram-se todos os minimizadores (locais) de $d$, os seus maximizadores (locais) e os possíveis pontos de sela dessa função.

Neste trabalho o principal interesse é encontrar todos os pontos estacionários da função distância e realizar uma análise sobre quais valores de parâmetros são os mais eficientes no desempenho do método Enxame de Partículas.

\section{O MÉTODO ENXAME DE PARTÍCULAS}

O método de otimização por Enxame de Partículas (Particle Swarm Optimization PSO) é uma metaheurística populacional baseada no movimento e comportamento coletivo de um sistema social. Trata-se de um método de otimização estocástica desenvolvido por Eberhart e Kennedy (1995) fundamentado em uma população, o enxame de $s$ partículas.

Seguindo a versão do PSO apresentada por Viot (2010), a posição de cada partícula é atualizada conforme as equações representadas por: 
Sendo:

$$
\begin{array}{r}
v_{i+1}=v_{i}+2 r n d()\left(p_{b e s t}-x_{i}\right)+2 r n d()\left(g_{\text {best }}-x_{i}\right)(0) \\
x_{i+1}=x_{i}+v_{i+1}(0)
\end{array}
$$

$x_{i}=$ posição da partícula.

$v_{i}=$ velocidade da partícula.

rnd ()$=$ número gerado aleatoriamente no intervalo $[0,1]$.

$p_{\text {best }}=$ melhor posição ocupada pela partícula.

$g_{\text {best }}=$ melhor posição ocupada pelo grupo.

$i=$ iteração.

Uma modificação no algoritmo inicial foi proposta por Eberhart e Shi (1998), introduzindo o peso inercial $w$ para controlar a velocidade entre as iterações das partículas e dois coeficientes de aceleração, parâmetro cognitivo $c_{1}$ e parâmetro social $c_{2}$, que objetivam ajudar o método a encontrar a solução ótima de modo mais rápido. Estes dois parâmetros na versão anterior eram fixos no valor 2. Conforme a modificação, as fórmulas se apresentam como:

Sendo:

$$
\begin{array}{r}
v_{i+1}=w v_{i}+c_{1} r n d()\left(p_{\text {best }}-x_{i}\right)+c_{2} r n d()\left(g_{\text {best }}-x_{i}\right)(0) \\
x_{i+1}=x_{i}+v_{i+1}(0)
\end{array}
$$

$w=$ peso inercial.

$c_{1}=$ coeficiente de aceleração cognitivo.

$c_{2}=$ coeficiente de aceleração social.

A seguir é apresentado o algoritmo do método PSO.

Algoritmo: Método Enxame de Partículas

1 Dados $c_{1}, c_{2}, w, n, d$.

2 Gerar aleatoriamente as posições das partículas do enxame $x_{0}^{1}, \ldots, x_{0}^{s}$ e os vetores que descrevem as velocidades iniciais $v_{0}^{1}, \ldots, v_{0}^{s}$.

3 Considere

$4 \quad p_{\text {best }}=x_{0}^{i} \forall i=1, \ldots, d$.

$5 \quad g_{\text {best }}=\min \left\{p_{\text {best }}^{1}, \ldots, p_{\text {best }}^{2}\right\}$

6 enquanto (critério de parada não for aceito) faça

$7 \quad$ para $i=1, \ldots, n$ faça

$8 \quad$ Atualizar a velocidade $v_{i+1}$ das partículas com a Eq. (7).

$9 \quad$ Atualizar a posição $x_{i+1}$ das partículas com a Eq. (8).

10 fim

$11 \quad$ Atualizar $p_{\text {best }}$ e $g_{\text {best }}$.

12 fim

\section{METODOLOGIA PROPOSTA}

A metodologia proposta para resolver o problema do teste de estabilidade consiste na utilização do método estocástico Enxame de Partículas. O problema exige que todos os minimizadores da função objetivo sejam encontrados e para isto será utilizada a técnica de polarização. Serão realizados testes no método numérico para analisar quais composições de seus parâmetros são mais eficientes para seu desempenho. 


\subsection{A TÉCNICA DA POLARIZAÇÃo}

A técnica da polarização é uma tática que auxilia na determinação de mais de um minimizador global da função distância, ou seja, permite encontrar mais de um ponto estacionário da função não negativa do problema.

Supondo que o primeiro minimizador global da função, denotado por $y^{(1)}$, foi determinado pelo método de otimização emprega-se o mesmo algoritmo de otimização para determinar o segundo minimizador global $\mathrm{y}^{(2)}$ resolvendo o problema (P2).

$\left\{\begin{array}{l}\operatorname{Minimizar} f_{1}(y)=\frac{f(y)+\alpha}{\operatorname{arctg}\left\|y-y^{(1)}\right\|} \\ y \in R^{r}, \alpha \geq 0\end{array}\right.$

Assim, de modo geral deve ser feito o mesmo processo de resolução dos subproblemas repetidas vezes para que todas as soluções sejam encontradas.

No processo de polarização procuram-se os pólos de maneira que supondo já encontradas $n>1$ soluções, busca-se a (n+1)-ésima solução resolvendo o problema (P3) a seguir.

$\left\{\begin{array}{l}\text { Minimizar } f_{n}(y)=\frac{f_{n-1}(y)}{\operatorname{arctg}\left\|y-y^{(n)}\right\|} \\ y \in R^{r}\end{array}\right.$

\subsection{Estudo de Parâmetros do Método EnXame de Partículas}

O objetivo foi realizar testes com estratégias de parâmetros aplicados ao problema de estabilidade para constatar os valores mais apropriados para o peso inercial $(w)$, parâmetro cognitivo $\left(c_{1}\right)$ e parâmetro social $\left(c_{2}\right)$.

Em 1995, Kennedy e Eberhart propuseram o algoritmo clássico do método que fixava os parâmetros $c_{1}$ e $c_{2}$ para o valor 2, para que desta forma, o fator randômico tivesse a média igual a 1. O algoritmo básico foi aprimorado em 1998 por Eberhart e Shi que introduziram a constante de inércia $w$ para atuar como o controlador da velocidade e as constantes de aceleração $c_{1}$ e $c_{2}$.

Segundo Mendes (2004), utilizar o coeficiente de inércia igual a 0,5 é favorável para a convergência do algoritmo assim, a primeira estratégia de valoração de parâmetros vai utilizar esta ideia juntamente com os mesmos coeficientes de aceleração do algoritmo fixado em 2.

Para a segunda estratégia de parâmetros, é preservado o valor $w=0,5$ e utilizada a ideia de Carlise e Dozier (2001), onde é assegurado que os parâmetros de aceleração são mais eficientes quando são utilizados valores aos quais $c_{1}>c_{2}$ e $c_{1}+c_{2} \leq 4$.

Visando a melhoria no desempenho do método, Eberhart e Shi (2000) recomendaram que no intervalo $[0,40,9]$ o valor de $w$ estivesse em uma variação linear conforme a fórmula (9). A terceira valoração de parâmetros emprega a proposta da variação linear da inércia.

$$
w=\left(w_{\text {inicial }}-w_{\text {final }}\right)\left[\frac{N-i}{i}\right]+w_{\text {final }}(0)
$$

Sendo:

$N$ - número total de iterações;

i - iteração atual; 
$w_{\text {inicial }}$ - valor inicial do peso de inércia;

$w_{\text {final }}$ - valor final do peso de inércia.

Chatterjee e Siarry (2006) aperfeiçoaram o conceito da variação linear da inércia para a variação não linear da inércia. Para a quarta estratégia de valoração de parâmetros utiliza-se a variação não linear da inércia, seguindo a fórmula (10), onde os valores $w_{\text {inicial }} \mathrm{e}$ $w_{\text {final }}$ são mantidos e ao longo das iterações $w$ pode ser controlado através do expoente de não linearidade $n$. A exemplo de Matias (2008), foi utilizado o valor 1,2 para o expoente de não linearidade.

$$
w=\left[\frac{(N-i)^{n}}{N^{n}}\right]\left(w_{\text {inicial }}-w_{\text {final }}\right)+w_{\text {final }}(0)
$$

Na quinta estratégia de valoração de parâmetros do presente estudo é utilizado o princípio do Coeficiente de Constrição. Clerc (1999) propôs um limitador para a velocidade inercial chamado de Fator de Constrição, este delimita $c_{1}$ e $c_{2}$ em função da velocidade.

$\left\{v_{i+1}=k\left[v_{i}+2 r n d()\left(p_{\text {best }}-x_{i}\right)+2 r n d()\left(g_{\text {best }}-x_{i}\right)\right] x_{i+1}=x_{i}+v_{i+1}(0)\right.$

$$
k=\frac{2}{\left|2-\varphi-\sqrt{\left(\varphi^{2}-4 \varphi\right)}\right|}(0)
$$

Onde:

$k \in[0,1]$;

$\varphi=c_{1}+c_{2}$;

$\varphi>4$;

$N$ - número total de iterações;

$i$ - iteração atual;

$w_{\text {inicial }}$ - valor inicial do peso de inércia;

$w_{\text {final }}$ - valor final do peso de inércia.

Seguindo Viot (2010) utiliza-se o valor de $\varphi=4,1, c_{1}=c_{2}=\frac{\varphi}{2}=2,05$, e $\mathrm{k}=0,729$.

\section{RESULTADOS NUMÉRICOS}

Nesta seção apresentam-se os resultados obtidos para as misturas com a metodologia proposta. Os testes foram realizados na função do problema termodinâmico. Para comparação foi utilizado o mesmo gerador de números aleatórios, para todas as estratégias de parâmetros.

\subsection{Resultados do Estudo dos Parâmetros}

O método PSO foi testado para três misturas binárias visando encontrar todos os pontos estacionários da função distância do teste de estabilidade. Os pontos estacionários foram obtidos através da técnica da polarização que é a estratégia que auxilia a encontrar todos os minimizadores globais da função objetivo. Os pontos mínimos globais das misturas foram comparados com os resultados encontrados por Oliveira (2016), utilizando o método estocástico Luus Jaakola, com o objetivo de validar os resultados obtidos pela metodologia proposta.

Os testes foram realizados em um Notebook Acer, munido de um processador Intel Core i3 com memória RAM de 4GB DDR4 e a implementação do método foi feita no 
Software Matlab. O programa foi executado 50 vezes e o resultado final é a média aritmética dos valores encontrados.

Os parâmetros do método PSO aplicados na função do problema são apresentados na Tabela 1, sendo avaliados em todas as misturas propostas.

Foram testadas as seguintes misturas binárias: n-pentanol /dimetilbutano, n-pentanol / metilpentano e a mistura etanol /ciclohexano.

Tabela 1: Parâmetros utilizados nas misturas binárias.

\begin{tabular}{c|c|c|c|}
\hline \multicolumn{4}{|c|}{ Parâmetros PSO } \\
\hline Dimensão & $\begin{array}{c}\text { Número de } \\
\text { Partículas }\end{array}$ & $\begin{array}{c}\text { Número } \\
\text { Máximo de } \\
\text { Execuções }\end{array}$ & Tolerância \\
\hline 2 & 30 & 100 & $10^{-6}$ \\
\hline
\end{tabular}

Fonte: A autora.

Os valores dos coeficientes a serem utilizados em cada uma das estratégias de valorações de parâmetros objetivados são apresentados nas Tabelas 2 e 3.

Tabela 2: Valores dos coeficientes.

\begin{tabular}{c|c|c|c}
\hline $\begin{array}{c}\text { Conjunt } \\
0\end{array}$ & $\mathrm{~W}$ & $\mathrm{c}_{1}$ & $\mathrm{c}_{2}$ \\
\hline 1 & 0,5 & 2 & 2 \\
\hline 2 & 0,5 & 2,5 & 1,5 \\
\hline 3 & Variação linear & 2 & 2 \\
\hline 4 & Variação não-linear & 2 & 2 \\
\hline 5 & 0,5 & 2,05 & 2,05 \\
\hline
\end{tabular}

Fonte: A autora.

Tabela 3: Dados da variação linear e não linear.

\begin{tabular}{c|c|c}
\hline & Variação linear & $\begin{array}{c}\text { Variação não } \\
\text { linear }\end{array}$ \\
\hline$w_{\text {inicial }}$ & 0,9 & 0,9 \\
\hline$w_{\text {final }}$ & 0,4 & 0,4 \\
\hline
\end{tabular}

Fonte: A autora.

A Tabela 4 expõe os resultados obtidos para a primeira mistura testada. Foram utilizados os parâmetros da Tabela 1 e os coeficientes dos conjuntos de parâmetros apresentados nas tabelas 2 e 3 .

Tabela 4: Resultados dos conjuntos de parâmetros na mistura: n-pentanol (1) / 2,2 dimetilbutano (2) - PSO. 
Fonte: A autora.

Observa-se que o método encontrou todos os mínimos globais da função. Analisando os dados da Tabela 4, nota-se que os conjuntos 3 e 4 foram os que melhor otimizaram as alimentações dadas a função objetivo, localizando os pontos estacionários com menores tempos e baixos números de iterações.

Os dados apresentados na Tabela 5 referem-se à mistura n-pentanol (1) / 2metilpentano (2).

É possível analisar a partir dos dados da Tabela 5 que o conjunto 3 foi a estratégia que obteve os resultados no menor tempo e baixos números de iterações. O conjunto 4 também destacou-se em relação ao tempo e ao número de iterações.

\begin{tabular}{|c|c|c|c|c|c|c|c|c|c|c|c|}
\hline \multirow[t]{2}{*}{ Alimentação } & \multirow{2}{*}{$\begin{array}{c}\text { Pontos } \\
\text { Estacionários }\end{array}$} & \multicolumn{2}{|c|}{ Conjunto 1} & \multicolumn{2}{|c|}{ Conjunto 2} & \multicolumn{2}{|c|}{ Conjunto 3} & \multicolumn{2}{|c|}{ Conjunto 4} & \multicolumn{2}{|c|}{ Conjunto 5} \\
\hline & & $\begin{array}{l}\text { Tempo } \\
\text { (s) }\end{array}$ & It. & $\begin{array}{c}\text { Tempo } \\
\text { (s) }\end{array}$ & It. & $\begin{array}{l}\text { Tempo } \\
\text { (s) }\end{array}$ & It. & $\begin{array}{l}\text { Tempo } \\
\text { (s) }\end{array}$ & It. & $\begin{array}{c}\text { Tempo } \\
\text { (s) }\end{array}$ & It. \\
\hline \multirow[t]{2}{*}{$\left(\begin{array}{ll}0.05 & 0.95\end{array}\right)$} & $\left(\begin{array}{ll}0.0500 & 0.9500\end{array}\right)$ & 0.2313 & 45 & 0.2367 & 54 & 0.3762 & 47 & 0.3502 & 62 & 0.2874 & 48 \\
\hline & $(0.1326 \quad 0.8669)$ & 0.3174 & 75 & 0.2584 & 59 & 0.2416 & 50 & 0.2913 & 47 & 0.3336 & 53 \\
\hline \multirow[t]{3}{*}{$\left(\begin{array}{ll}0.10 & 0.90\end{array}\right)$} & $\left(\begin{array}{lll}0.1000 & 0.9000\end{array}\right)$ & 0.2781 & 71 & 0.2199 & 54 & 0.1857 & 45 & 0.1507 & 33 & 0.2814 & 66 \\
\hline & $\left(\begin{array}{ll}0.0692 & 0.9308\end{array}\right)$ & 0.3099 & 66 & 0.1921 & 38 & 0.2721 & 51 & 0.2758 & 52 & 0.3025 & 68 \\
\hline & $\left(\begin{array}{ll}0.1500 & 0.8500\end{array}\right)$ & 0.2440 & 52 & 0.2201 & 46 & 0.3016 & 65 & 0.2052 & 44 & 0.3160 & 70 \\
\hline \multirow[t]{3}{*}{$\left(\begin{array}{ll}0.15 & 0.85\end{array}\right)$} & $\left.\begin{array}{ll}0.1500 & 0.8500\end{array}\right)$ & 0.2734 & 69 & 0.2421 & 60 & 0.2281 & 50 & 0.1907 & 45 & 0.3027 & 72 \\
\hline & $\left(\begin{array}{ll}0.0692 & 0.9308\end{array}\right)$ & 0.3773 & 91 & 0.3132 & 68 & 0.2191 & 47 & 0.2341 & 38 & 0.2571 & 55 \\
\hline & $\left(\begin{array}{ll}0.1000 & 0.9000\end{array}\right)$ & 0.3822 & 87 & 0.2919 & 62 & 0.2887 & 59 & 0.2628 & 57 & 0.2278 & 48 \\
\hline \multirow[t]{3}{*}{$\left.\begin{array}{ll}0.20 & 0.80\end{array}\right)$} & $\left.\begin{array}{ll}(0.2000 & 0.8000\end{array}\right)$ & 0.2301 & 60 & 0.2243 & 54 & 0.1881 & 28 & 0.1836 & 43 & 0.3499 & 90 \\
\hline & $\left(\begin{array}{ll}0.0768 & 0.9245\end{array}\right)$ & 0.2157 & 47 & 0.2300 & 50 & 0.2098 & 42 & 0.2862 & 68 & 0.3113 & 73 \\
\hline & $\left(\begin{array}{ll}0.0771 & 0.9242\end{array}\right)$ & 0.2490 & 51 & 0.3912 & 76 & 0.2081 & 40 & 0.2341 & 50 & 0.2427 & 55 \\
\hline
\end{tabular}

Resultados dos conjuntos de parâmetros na mistura: n-pentanol (1) / 2-metilpentano (2) PSO

\begin{tabular}{|c|c|c|c|c|c|c|c|c|c|c|c|c|}
\hline \multirow[t]{2}{*}{ Alimentação } & \multirow{2}{*}{\multicolumn{2}{|c|}{$\begin{array}{c}\text { Pontos } \\
\text { Estacionários }\end{array}$}} & \multicolumn{2}{|c|}{ Conjunto 1} & \multicolumn{2}{|c|}{ Conjunto 2} & \multicolumn{2}{|c|}{ Conjunto 3} & \multicolumn{2}{|c|}{ Conjunto 4} & \multicolumn{2}{|c|}{ Conjunto 5} \\
\hline & & & $\begin{array}{c}\text { Tempo } \\
\text { (s) }\end{array}$ & It. & $\begin{array}{l}\text { Tempo } \\
\text { (s) }\end{array}$ & It. & $\begin{array}{c}\text { Tempo } \\
\text { (s) }\end{array}$ & It. & $\begin{array}{c}\text { Tempo } \\
\text { (s) }\end{array}$ & It. & $\begin{array}{c}\text { Tempo } \\
\text { (s) }\end{array}$ & It. \\
\hline \multirow[t]{2}{*}{$\left(\begin{array}{ll}0.05 & 0.95\end{array}\right)$} & $(0.0500$ & $0.9500)$ & 0.3745 & $\begin{array}{c}10 \\
1\end{array}$ & 0.2895 & 74 & 0.2191 & 45 & 0.2522 & 47 & 0.2328 & 61 \\
\hline & $(0.1397$ & $0.8599)$ & 0.2604 & 58 & 0.3220 & 77 & 0.2268 & 48 & 0.4218 & 51 & 0.2515 & 49 \\
\hline \multirow[t]{3}{*}{$\left(\begin{array}{ll}0.10 & 0.90\end{array}\right)$} & $(0.1000$ & $0.9000)$ & 0.2614 & 64 & 0.2270 & 55 & 0.2787 & 64 & 0.2204 & 49 & 0.3095 & 46 \\
\hline & $(0.1657$ & $0.8343)$ & 0.2322 & 53 & 0.4004 & 53 & 0.1856 & 43 & 0.1939 & 39 & 0.1135 & 23 \\
\hline & $(0.0688$ & $0.9312)$ & 0.4038 & 74 & 0.2376 & 49 & 0.2524 & 54 & 0.1598 & 29 & 0.3097 & 70 \\
\hline \multirow[t]{3}{*}{$\left(\begin{array}{lll}0.11 & 0.89\end{array}\right)$} & $(0.1100$ & $0.8900)$ & 0.2733 & 48 & 0.3212 & 71 & 0.1829 & 45 & 0.1814 & 45 & 0.1915 & 47 \\
\hline & (0.1582 & $0.8418)$ & 0.5340 & 64 & 0.2251 & 51 & 0.1952 & 45 & 0.3357 & 57 & 0.2183 & 44 \\
\hline & $(0.0654$ & $0.9346)$ & 0.3635 & 79 & 0.2163 & 44 & 0.2422 & 54 & 0.2106 & 43 & 0.1753 & 37 \\
\hline \multirow[t]{3}{*}{$\left(\begin{array}{ll}0.12 & 0.88\end{array}\right)$} & $(0.1200$ & $0.8800)$ & 0.2613 & 66 & 0.1331 & 29 & 0.2038 & 49 & 0.1816 & 46 & 0.1921 & 49 \\
\hline & (0.1495 & 0.8505 & 0.3308 & 78 & 0.2494 & 61 & 0.2170 & 50 & 0.2021 & 47 & 0.1893 & 43 \\
\hline & $(0.0633$ & $0.9367)$ & 0.2180 & 46 & 0.3245 & 73 & 0.3238 & 59 & 0.2277 & 49 & 0.2702 & 61 \\
\hline \multirow[t]{3}{*}{$\left(\begin{array}{ll}0.20 & 0.80\end{array}\right)$} & $(0.2000$ & $0.8000)$ & 0.1849 & 44 & 0.2231 & 57 & 0.1487 & 34 & 0.1688 & 41 & 0.1849 & 46 \\
\hline & (0.0808 & 0.91 & 0.4075 & 76 & 0.2667 & 63 & 0.2406 & 52 & 0.2684 & 57 & 0.2692 & 66 \\
\hline & $(0.0731$ & $0.9291)$ & 0.1833 & 37 & 0.2479 & 55 & 0.1831 & 38 & 0.1854 & 39 & 0.1976 & 42 \\
\hline \multirow[t]{3}{*}{ (0.25 0.75) } & $(0.2500$ & $0.7500)$ & 0.3012 & 77 & 0.3296 & 48 & 0.2402 & 44 & 0.2060 & 53 & 0.2442 & 36 \\
\hline & (0.0782 & 0.92 & 0.2567 & 54 & 0.3 & 72 & 0.2754 & 50 & 0.1826 & 35 & 0.2263 & 50 \\
\hline & $(0.0779$ & $0.9248)$ & 0.2405 & 51 & 0.2535 & 56 & 0.1975 & 43 & 0.1920 & 41 & 0.0044 & 41 \\
\hline
\end{tabular}

Fonte: A autora.

A tabela 6 a seguir, apresenta os resultados encontrados para a mistura etanol (1)/ciclohexano (2). 
Tabela 6: Resultados dos conjuntos de parâmetros na mistura: Etanol 1 / ciclohexano 2 PSO

\begin{tabular}{|c|c|c|c|c|c|c|c|c|c|c|c|}
\hline \multirow{2}{*}{$\begin{array}{c}\text { Alimentaçã } \\
\text { o }\end{array}$} & \multirow{2}{*}{$\begin{array}{c}\text { Pontos } \\
\text { Estacionários }\end{array}$} & \multicolumn{2}{|c|}{ Conjunto 1} & \multicolumn{2}{|c|}{ Conjunto 2} & \multicolumn{2}{|c|}{ Conjunto 3} & \multicolumn{2}{|c|}{ Conjunto 4} & \multicolumn{2}{|c|}{ Conjunto 5} \\
\hline & & $\begin{array}{c}\text { Tempo } \\
\text { (s) }\end{array}$ & It. & $\begin{array}{c}\text { Tempo } \\
\text { (s) }\end{array}$ & It. & $\begin{array}{c}\text { Tempo } \\
\text { (s) }\end{array}$ & It. & $\begin{array}{c}\text { Tempo } \\
\text { (s) }\end{array}$ & It. & $\begin{array}{c}\text { Tempo } \\
\text { (s) }\end{array}$ & It. \\
\hline \multirow[t]{3}{*}{$\left.\begin{array}{ll}0.05 & 0.95\end{array}\right)$} & $\begin{array}{lll}0.0500 & 0.9500\end{array}$ & 0.4226 & 51 & 0.2492 & 56 & 0.1957 & 45 & 0.1972 & 32 & 0.3328 & 62 \\
\hline & (0.0942 0.9058$)$ & 0.3629 & 72 & 0.2730 & 54 & 0.2328 & 42 & 0.3017 & 49 & 0.4415 & 94 \\
\hline & $\left(\begin{array}{ll}0.5979 & 0.4021\end{array}\right)$ & 0.3466 & 67 & 0.2671 & 51 & 0.2511 & 43 & 0.2331 & 42 & 0.3232 & 62 \\
\hline \multirow[t]{3}{*}{$\left.\begin{array}{ll}0.10 & 0.90\end{array}\right)$} & $\begin{array}{lll}0.1000 & 0.9000)\end{array}$ & 0.2336 & 45 & 0.2432 & 47 & 0.2475 & 54 & 0.2224 & 50 & 0.3314 & 67 \\
\hline & $\left(\begin{array}{lll}0.0475 & 0.9529\end{array}\right)$ & 0.4228 & 67 & 0.2146 & 45 & 0.3550 & 64 & 0.2770 & 57 & 0.4082 & 83 \\
\hline & $\left(\begin{array}{ll}0.5878 & 0.4122\end{array}\right)$ & 0.3506 & 68 & 0.2682 & 57 & 0.2122 & 41 & 0.2172 & 42 & 0.3508 & 62 \\
\hline \multirow[t]{3}{*}{ (0.15 0.85) } & $\begin{array}{lll}0.1500 & 0.8500\end{array}$ & 0.3668 & 88 & 0.2201 & 53 & 0.4628 & 52 & 0.2150 & 49 & 0.2861 & 60 \\
\hline & $\left(\begin{array}{ll}0.0352 & 0.9659\end{array}\right)$ & 0.5914 & $\begin{array}{c}11 \\
3\end{array}$ & 0.3574 & 77 & 0.3330 & 61 & 0.2877 & 53 & 0.2009 & 32 \\
\hline & $\left(\begin{array}{ll}0.4880 & 0.5120\end{array}\right)$ & 0.2869 & 56 & 0.3035 & 58 & 0.2530 & 52 & 0.2150 & 41 & 0.2530 & 50 \\
\hline \multirow[t]{3}{*}{ 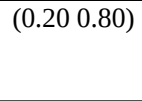 } & $\left(\begin{array}{lll}0.2000 & 0.8000\end{array}\right)$ & 0.2936 & 62 & 0.3214 & 66 & 0.2166 & 51 & 0.2112 & 46 & 0.2680 & 65 \\
\hline & $\left(\begin{array}{ll}0.4000 & 0.6000\end{array}\right)$ & 0.2670 & 53 & 0.2905 & 49 & 0.2026 & 45 & 0.2290 & 44 & 0.3166 & 70 \\
\hline & 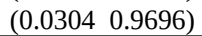 & 0.4265 & 87 & 0.3281 & 61 & 0.1624 & 30 & 0.2583 & 48 & 4.3359 & 1000 \\
\hline \multirow{3}{*}{$\left(\begin{array}{lll}0.25 & 0.75)\end{array}\right.$} & $\begin{array}{lll}0.2500 & 0.7500\end{array}$ & 0.3307 & 81 & 0.3199 & 55 & 0.2443 & 38 & 0.2403 & 45 & 0.3960 & 62 \\
\hline & (0.3314 0.6686$)$ & 0.3097 & 72 & 0.2446 & 49 & 0.1859 & 41 & 0.2192 & 47 & 0.3194 & 72 \\
\hline & $\left(\begin{array}{ll}0.0285 & 0.9715\end{array}\right)$ & 0.1990 & 36 & 0.2955 & 64 & 0.2707 & 56 & 0.3764 & 56 & 0.3226 & 59 \\
\hline \multirow[t]{3}{*}{$(0.350 .65)$} & $\left(\begin{array}{ll}0.3500 & 0.6500\end{array}\right)$ & 0.3339 & 73 & 0.2592 & 55 & 0.1690 & 37 & 0.2215 & 55 & 0.3234 & 80 \\
\hline & (0.2351 0.7649$)$ & 0.2958 & 65 & 0.2782 & 53 & 0.2000 & 44 & 0.1343 & 25 & 0.2686 & 62 \\
\hline & $\left(\begin{array}{lll}0.0287 & 0.9678\end{array}\right)$ & 0.2852 & 60 & 0.3816 & 76 & 0.1667 & 32 & 0.2782 & 54 & 0.3821 & 89 \\
\hline \multirow[t]{3}{*}{$\left(\begin{array}{l}0.40 \\
0.60)\end{array}\right.$} & $\begin{array}{lll}0.4000 & 0.6000\end{array}$ & 0.2114 & 52 & 0.2731 & 60 & 0.2153 & 44 & 0.2204 & 54 & 0.2435 & 61 \\
\hline & $\left(\begin{array}{ll}0.2000 & 0.8000\end{array}\right)$ & 0.2702 & 62 & 0.2876 & 61 & 0.2293 & 47 & 0.2180 & 50 & 0.2985 & 71 \\
\hline & $\left(\begin{array}{lll}0.0304 & 0.9697\end{array}\right)$ & 0.4301 & 97 & 0.3031 & 59 & 0.4420 & 89 & 0.2588 & 53 & 0.2405 & 50 \\
\hline \multirow[t]{3}{*}{$(0.450 .55)$} & $\left(\begin{array}{ll}0.4500 & 0.5500\end{array}\right)$ & 0.3705 & 59 & 0.2676 & 59 & 0.2349 & 50 & 0.1804 & 44 & 0.4489 & 66 \\
\hline & $\left(\begin{array}{lll}0.0327 & 0.9673\end{array}\right)$ & 0.3169 & 69 & 0.2917 & 57 & 0.2297 & 39 & 0.3394 & 74 & 0.3275 & 72 \\
\hline & $\left(\begin{array}{ll}0.1702 & 0.8298\end{array}\right)$ & 0.2819 & 60 & 0.2601 & 48 & 0.2713 & 54 & 0.2578 & 50 & 0.3498 & 58 \\
\hline \multirow[t]{3}{*}{$\left(\begin{array}{lll}0.60 & 0.40\end{array}\right)$} & $\left(\begin{array}{ll}0.6000 & 0.4000\end{array}\right)$ & 0.2550 & 57 & 0.2763 & 61 & 0.2095 & 35 & 0.2065 & 48 & 0.1851 & 45 \\
\hline & $\left(\begin{array}{ll}0.0506 & 0.9495\end{array}\right)$ & 0.4841 & $\begin{array}{c}10 \\
0\end{array}$ & 0.2799 & 53 & 0.3059 & 58 & 0.3633 & 64 & 4.3762 & 1000 \\
\hline & $\left(\begin{array}{ll}0.0929 & 0.9071\end{array}\right)$ & 0.2409 & 54 & 0.3017 & 58 & 0.4775 & 93 & 0.2790 & 52 & 0.3002 & 64 \\
\hline \multirow[t]{2}{*}{ (0.65 0.35$)$} & $\left(\begin{array}{ll}0.6500 & 0.3500\end{array}\right)$ & 0.3281 & 45 & 0.3065 & 61 & 0.2196 & 46 & 0.1688 & 41 & 0.4372 & 63 \\
\hline & $\left(\begin{array}{ll}0.0679 & 0.9321)\end{array}\right.$ & 0.2488 & 49 & 0.2416 & 45 & 0.3262 & 63 & 0.3504 & 78 & 0.5707 & 107 \\
\hline
\end{tabular}

Fonte: A autora.

Nos dados da Tabela 6, nota-se que ao realizar os testes para a terceira mistura abordada, pode-se afirmar que a estratégia do conjunto 4 foi a que apresentou as soluções que melhor convergiram a função apresentando baixo tempo e bons números de iterações. $\mathrm{O}$ conjunto 3 também conseguiu bons resultados. Cabe destacar que para esta mistura, o conjunto 5 foi o que encontrou a maior dificuldade na minimização, visto que teve maiores tempos nas mais altas iterações.

\section{CONCLUSÕES}

Analisando o estudo realizado neste trabalho é possível afirmar que o Método Enxame de Partículas foi eficiente e robusto na minimização do problema. O método apresentou um bom desempenho e encontrou todos os pontos estacionários da função distância. De acordo com os testes numéricos realizados, identificou-se que utilizando as técnicas da variação linear e também da variação não linear da inércia no método de otimização, para o problema abordado, garantiu boa eficiência temporal na busca pelos mínimos globais. Pode-se concluir que a metodologia proposta foi um bom instrumento para se verificar qual estratégia de parâmetros atuam melhor no método aplicado ao problema da análise de estabilidade de fases de uma mistura. 


\section{SPOLM2019}

XIX SIMPÓSIO DE PESQUISA OPERACIONAL ELOGÍSTICA DA MARINHA

\section{AGRADECIMENTOS}

Agradeço a Fundação de Amparo à Pesquisa do Estado do Rio de Janeiro (FAPERJ), pelo apoio financeiro a este projeto de pesquisa.

\section{REFERÊNCIAS BIBLIOGRÁFICAS}

[1] CARLISLE, A., DOZIER, G., An Off-The-Shelf PSO. Proceedings of the Particle Swarm, Optimization Workshop, pp. 1-6, 2001.

[2] CHATTERJEE, A., SIARRY, P. Nonlinear Inertia Weight Variation for Dynamic Adaptation in Particle Swarm Optimization, Computers \& Operational Research, Volume 33, Issue 3, pp 859-871, 2006.

[3] CLERC, M. The swarm and the queen: towards a deterministic and adaptive particle swarm optimization. Proc. I999 ICEC, Washington, DC, p. 1951-1957, 1999.

[4] EBERHART, R. C.; SHI, Y. Particle Swarm Optimization: Developments, Applications and Resources. In: IEEE Congr. Evol. Comput., vol. 1,2001. p. 81-86.

[5] KENNEDY, J.; EBERHART, R. Particle swarm optimization. In: IEEE INTERNATIONAL CONFERENCE ON NEURAL NETWORKS, 1995, Perth. Proceedings ... Piscataway, NJ: IEEE Service Center, 1995. p 1942-1948.

[6] LUCIA, A., DiMAgGiO, P. A., BELlOWS, M. L. e OCTAVIO, L. M. The phase behavior of n-alkane systems. Computers \& chemical engineering, v. 29, n. 11-12, p. 2363-2379, 2005.

[7] MATIAS, P.T. Avaliação Comparativa de Algoritmos evolutivos com operadores adaptativos. Rio de Janeiro, 2008. 56 p. Dissertação (Mestrado em Ciência em Engenharia Civil) - COPPE, Universidade Federal do Rio de Janeiro, Rio de Janeiro, 2008. Disponível em: < http://www.coc.ufrj.br/index.php? option=com_docman\&task=doc_details\&gid=1457 > . Acesso em: 04 agosto 2018 .

[8] MENDES, R. Population Topologies and Their Influence in Particle Swarm Performance. 2004. 189 p. Tese (Doutorado)- Departamento de Informática, Escola de Engenharia, Universidade do Minho, 1994. Disponível em: < http://citeseerx.ist.psu.edu/viewdoc/download? doi=10.1.1.150.6463\&rep=rep1\&type=p df $>$. Acesso: 10 agosto 2018.

[9] MICHELSEN, M. L. The Isothermal Flash Problem. Part I - Stability Analysis. Part II - Phase Split Calculation. Fluid Phase Equilibria, v. 9, p. 1-19, 21-40, 1982.

[10] OLIVEIRA, M. B. Aplicação do Método Luus Jaakola em um Problema Termodinâmico. Santo Antônio de Pádua, 2016. 67 p. Trabalho de Conclusão de Curso (Graduação em Matemática) - Instituto do Noroeste Fluminense de Educação Superior, Universidade Federal Fluminense, Santo Antônio de Pádua, 2016.

[11] SOUZA, J. S. Análise global da estabilidade termodinâmica de misturas: um estudo com o método do conjunto gerador. Nova Friburgo, 2010. 138 f. Tese (Doutorado em Modelagem Computacional) - IPRJ, Universidade do Estado do Rio de Janeiro, Nova Friburgo- RJ, 2010. Disponível em:

$<$ http://www.bdtd.uerj.br/tde_busca/processaPesquisa.php?pesqExecutada=1\&id=1869 \&PHPSESSID=lpbth6m3objo1o7085flupu2n0>. Acesso em: 18 abril 2019. 
[12] STADTHERR, M. A., SCHNEPPER, C. A., BRENNECKE, J. F. Robust Phase Stability and Analysis using Interval Methods. In: AIChE Symposium Series. New York, NY: American Institute of Chemical Engineers, v. 91, n. 304, p. 356- 359, 1995.

[13] SUN, A. C., SEIDER, W. D. Homotopy-continuation method for stability analysis in the global minimization of the Gibbs free energy. Fluid Phase Equilibria, v. 103, n. 2, p. 213-249, 1995.

[14] VIOT, J. Otimização por Enxame de Partículas com congregação passiva seletiva. Rio de Janeiro, 2010. 94 p. Tese (Doutorado em ciências em engenharia civil) COPPE, Universidade Federal do Rio de Janeiro, Rio de Janeiro, 2010. Disponível em: $<\quad$ http://www.dominiopublico.gov.br/pesquisa/DetalheObraForm.do? select_action=\&co_obra=181377>. Acesso em: 08 agosto 2018. 